\title{
Facilitation of Olfactory Learning by a Modulator of AMPA Receptors
}

\author{
J. Larson, ${ }^{1}$ T. Lieu, ${ }^{1}$ V. Petchpradub, ${ }^{1}$ B. LeDuc, ${ }^{1}$ H. Ngo, ${ }^{1}$ G. A. Rogers,,${ }^{2, a}$ and G. Lynch \\ ${ }^{1}$ Center for the Neurobiology of Learning and Memory, University of California, Irvine, California 92717-3800 and \\ ${ }^{2}$ Neuroscience Research Institute, University of California, Santa Barbara, California 93106
}

The effects of a benzoyl-piperidine drug (BDP) that facilitates AMPA receptor-mediated synaptic responses were tested on the acquisition and retention of long-term memory at dosages that had no detectable effects on a variety of performance measures. BDP-12 produced a dose-dependent suppression of exploratory activity in rats with statistically reliable effects occurring at $50 \mathrm{mg} / \mathrm{kg}$ (i.p.). The drug had no effects on balance beam performance at $30 \mathrm{mg} / \mathrm{kg}$ but at $45 \mathrm{mg} / \mathrm{kg}$ reduced the number of crossings made within a session; it did not, however, affect the time required to perform a traversal. The performance of welltrained rats presented with a familiar pair of odors (correct and incorrect) was not detectably altered by BDP-12 at 30 $\mathrm{mg} / \mathrm{kg}$; however, the number of correct responses made in a five-trial test was reduced at $45 \mathrm{mg} / \mathrm{kg}$. These results indicate that the AMPA receptor modulator at $30 \mathrm{mg} / \mathrm{kg}$ has little influence on arousal, motivation, sensori-motor processing, and attention; higher dosages cause a depression of learned and unlearned prepotent responses. The effects of the lower concentration were tested on two-odor discrimination learning in rats that had extensive training on the task. The animals $(n=20)$ were given three or five acquisition trials with novel odor pairs immediately after an injection of drug or vehicle and then tested 1-3 d later for retention in five unrewarded probe trials. Retention performance was not significantly better than chance (52.6 \pm $4.5 \%$ correct) for odors learned on vehicle injection days but was well above chance for odors learned on drug injection days $(70.6 \pm 4.2 \%$ correct). Within-subject comparisons confirmed the memory enhancing effect of BDP-12 $(p<0.01)$. Analyses of performance during five training trials indicated that the rats made more correct responses on days on which they were given the drug than on days on which they were injected with vehicle $(p<0.02)$. Withinsubject differences in acquisition were correlated with differences in retention $(r=0.70)$. There were no evident effects of the drug on response latencies during acquisition. These results suggest that AMPA receptor modulators reduce the amount of training needed for the formation of long-term memory and do so at dosages which have little

\footnotetext{
Received Dec. 22, 1994; revised July 28, 1995; accepted Aug. 8, 1995.

This research was supported by grants from the Air Force Office of Scientific Research (AFOSR 92-J-0307, to G.L.) and the National Institute of Mental Health (MH-51151, to J.L.).

Correspondence should be addressed to Dr. John Larson at the above address.

apresent address: Cortex Pharmaceuticals, Inc., Irvine, CA 92718.

Copyright $\mathcal{O} 1995$ Society for Neuroscience $\quad 0270-6474 / 95 / 158023-08 \$ 05.00 / 0$
}

effect on variables that secondarily influence acquisition. Possible reasons for this selectivity are discussed.

[Key words: AMPA receptor, glutamate receptor, longterm potentiation, olfactory learning, acquisition, retention, exploratory activity, balance beam]

Induction of long-term potentiation (LTP) normally requires a spatial and temporal summation of fast, excitatory synaptic responses mediated by the AMPA subclass of glutamate receptors; this allows voltage-dependent NMDA receptors to admit calcium, the intracellular trigger for potentiation, into the postsynaptic cell (see Bliss and Collingridge, 1993, for review). Recent studies have identified drugs that facilitate postsynaptic currents generated by AMPA receptors and thus would be expected to promote the induction of LTP. Ito et al. (1990) found that the nootropic compound, aniracetam, enhances AMPA receptor-gated currents in oocytes and increases synaptic responses in hippocampal slices. Subsequent studies confirmed the prediction that aniracetam facilitates the induction of LTP; that is, the number of afferent bursts needed to elicit a near maximal level of potentiation was reduced by about $50 \%$ in the presence of the drug (Arai and Lynch, 1992). Given that there is considerable evidence linking LTP to the encoding of some types of memory, it was predicted that centrally active AMPA receptor modulators would have a measurable effect on acquisition and retention in a variety of learning paradigms.

These considerations led to the development of a class of benzoyl-piperidine (BDP) compounds that enhance AMPA receptor currents (Arai el al,, 1994), increase fast synaptic responses in hippocampus, and freely cross the blood-brain barrier after peripheral administration (Staubli et al., 1994a,b). These drugs were found to improve recent memory of spatial (Granger et al., 1993; Staubli et al., 1994a,b) and olfactory (Staubli et al., 1994b) cues as well as to enhance long-term retention in a variety of paradigms (Shors et al., 1994; Staubli et al., 1994a).

While the above results confirm a prediction of the hypothesis that LTP is a substrate of some types of memory, the observed effects of the BDP drugs could potentially be due to actions not directly related to encoding. Such possible actions include the following: (1) increases in arousal, motivation, or other state variables that secondarily influence acquisition, (2) changes in sensory/motor systems involved in the acquisition of pertinent cues and performance of appropriate responses, (3) enhancement of posttrial events involved in consolidation. The present studies investigated the relative effects of an AMPA receptor modulator on certain of the variables listed above. Specifically, different dosages of the drug were tested for their effects on measures that were sensitive to changes in arousal, motivation, sensori- 
motor integration, and response latency to pertinent cues. A drug concentration that had little or no effect on these measures was then used in a long-term memory paradigm which allowed for analysis of rate of acquisition as well as of the strength of retention.

An olfactory discrimination test was employed for the learning studies. After training on a series of several two-odor discriminations, rats can acquire new discriminations in as few as 5-10 trials (Slotnick and Katz, 1974; Eichenbaum et al., 1986; Staubli et al., 1987; Lu and Slotnick, 1990; Slotnick et al., 1991). They also learn dozens of odors and demonstrate retention of the significance of individual cues for weeks (Staubli et al., 1987; Slotnick and Kisser, 1990; Slotnick et al., 1991; Youngentob et al., 1991). Thus, odor discriminations are rapidly acquired, remembered for long periods without interference from other cues, and the storage system has a large capacity. This makes it possible to test for acquisition of different novel cues repeatedly over several days with different drug treatments as well as to test the strength of retention days after training. Furthermore, drug effects on performance of already-learned information can be assessed. These features allow for within-animal comparisons of drug versus vehicle effects on long-term memory and, more generally, are of use in analyzing how experimental compounds might influence the learning process.

The drug used for the present studies is an analog of 1-(1,3benzodioxol-5-ylcarbonyl) piperidine (BDP). The effects of this compound (BDP-12) on monosynaptic and polysynaptic responses have been extensively analyzed in hippocampal slices (Coogan et al., 1994; Sirvio et al., 1994); moreover, the drug has been reported to facilitate recent memory in radial mazes (Granger et al., 1993, in press) and to enhance the formation of conditioned fear responses (Rogan et al., 1994). These earlier findings provided a starting point for investigations of how AMPA receptor modulators interact with the stable encoding of discriminative cues.

\section{Materials and Methods}

\section{Spontaneous locomotor activity}

Twenty-nine Sprague-Dawley rats (Charles River Laboratories) about $60 \mathrm{~d}$ old and housed in groups of four per cage with food and water available ad libitum were used for tests of spontaneous activity in a novel environment. Rats were tested during the light phase of the light/ dark cycle (12:12, lights on at 7:00 A.M.). BDP-12 was dissolved in $33 \%$ (w:v) 2-hydroxypropyl- $\beta$-cyclodextrin vehicle in $0.45 \%$ saline. Ten minutes after a single intraperitoneal (i.p.) injection of $25 \mathrm{mg} / \mathrm{kg}(n=$ $4), 50 \mathrm{mg} / \mathrm{kg}(n=4), 75 \mathrm{mg} / \mathrm{kg}(n=7) \mathrm{BDP}-12$ or vehicle $(n=14)$, rats were placed individually into a clear plexiglas box $50 \mathrm{~cm}$ long $x$ $25 \mathrm{~cm}$ deep $\times 80 \mathrm{~cm}$ high. The box was inside a large, dimly lit wooden enclosure cquipped with an exhaust fan and a window for viewing. Each test was $15 \mathrm{~min}$. long and consisted of counting each crossing of the center line and each instance of rearing. Analysis of variance with planned comparisons were used to evaluate the results statistically.

\section{Drugs}

BDP- 12 was synthesized by G.A.R. and Cortex Pharmaceuticals, Irvine, CA. 2-Hydroxypropyl- $\beta$-cyclodextrin was purchased from Research Biochemicals, Natick, MA.

\section{Balance beam training}

\section{Subjects}

Eight Sprague-Dawley rats aged approximately $60 \mathrm{~d}$ at the outset of training served as subjects. The animals were housed individually and were on a 12:12 light:dark cycle. Testing was done during the light phase. During training, food was available ad libitum but access to water was restricted to that obtained during the training session and for a period of $10-15$ min afterward each day.

\section{Apparatus}

The balance beam was a $1 \mathrm{~m}$ long square wooden beam $2 \mathrm{~cm}$ wide elevated $1 \mathrm{~m}$ above a cushioned floor. At each end of the beam was a $30 \mathrm{~cm} \times 30 \mathrm{~cm}$ square horizontal platform containing a water dish.

\section{Procedure}

Initial training. After $5 \mathrm{~d}$ on the water deprivation schedule and daily handling, rats were placed on the center of the beam for 10 trials per day and allowed to cross the beam to obtain water rewards $(100 \mu \mathrm{l})$ on either platform. Each trial had a duration of 2 min. After the first traversal, water was available only on the platform opposite the rat's position. Rats were given 21 sessions at the end of which each rat would make several crossings of the beam to obtain water during each trial. They were then given a series of daily sessions consisting of one 10 min trial each day until performance stabilized (10 sessions).

Drug testing. Drug testing sessions were run in blocks of two consecutive days. Each session consisted of one $10 \mathrm{~min}$. trial heginning 10 min after i.p. injections. On day 1 of each block, four of the rats received BDP-12 injections and the other four received vehicle injections with treatment randomized. On day 2 , each rat received the treatment not received on day 1 . In between blocks the rats were tested on the beam without drug treatments. During drug blocks, behavior on the beam was videotaped and the number of beam traversals and the lime taken for each traversal were measured. Average traversal times for the first and second group of 10 crossings in each trial were calculated. Each rat was tested twice after $30 \mathrm{mg} / \mathrm{kg} \mathrm{BDP}-12$ and vehicle injections and twice after $50 \mathrm{mg} / \mathrm{kg}$ BDP-12 and vehicle injections; all tests being conducted on different days. Paired comparisons were made withinanimal.

\section{Olfactory discrimination learning}

\section{Subjects}

The subjects for this study were twenty male Sprague-Dawley rats of similar age and housing conditions as described for the animals tested on the balance beam. All training and testing was conducted during the light phase. Food was available ad libitum but access to water during the experiment was restricted to that obtained during training sessions and for a $10-15$ min period daily after training or testing.

\section{Apparatus}

All testing was conducted in a wedge-shaped enclosure described previously (Staubli et al., 1989) with a few modifications. The chamber consisted of a start box, a central arena and six alleys. At the end of each alley was a cylindrical sniff port $(2 \mathrm{~cm}$ diameter) equipped with an infrared photobeam detection system and through which odor stimuli could be delivered. Below each sniff port was a cup for water reinforceunent. Odor cues consisted of air (2 liter $/ \mathrm{min}$ ) blown through $125 \mathrm{ml}$ flasks containing 2-24 ml of concentrated odorant. Odors were selected at random from a stock of eighty spices, plant extracts, perfumes, and organic compounds (International Flavors and Fragrances, Schilling, McCormick). Flow of air through the odorizing flasks was regulated by a solenoid valve upstream from the flasks and was diverted between trials to a vacuum line. A computer controlled all trial events and recorded behavioral responses.

\section{Procedure}

Nose-poke training. Rats were initially trained to nose-poke in sniff ports in six daily sessions of 40 trials each. Each trial was preceded by a 5-sec. inter-trial interval (ITI) during which a lamp at the top of the chamber was illuminated and nose-pokes had no consequences. At the end of the ITI, the lamp was extinguished and any nose-poke into an alley different from the last reinforced alley was rewarded with water $(150 \mu 1)$. Either a rewarded nose-poke or the end of the $40 \mathrm{sec}$ trial period terminated the trial and initiated the next ITI.

Odor discrimination training. Rats were trained on the first two-odor discrimination in daily 20 trial sessions. Each trial was preceded by a 1 min ITI during which the rat was in its home cage. Positive and negative alleys were determined randomly for each trial subject to the constraint that no alley ever contained the same stimulus on consecutive trials. At the onset of a trial the rat was placed in the start box and the odorized air turned on. A nose-poke into the sniffport carrying the positive odor (correct response) was rewarded with water ( $150 \mu l)$; a nosepoke in the negative alley (incorrect response) triggered a flashing light. 

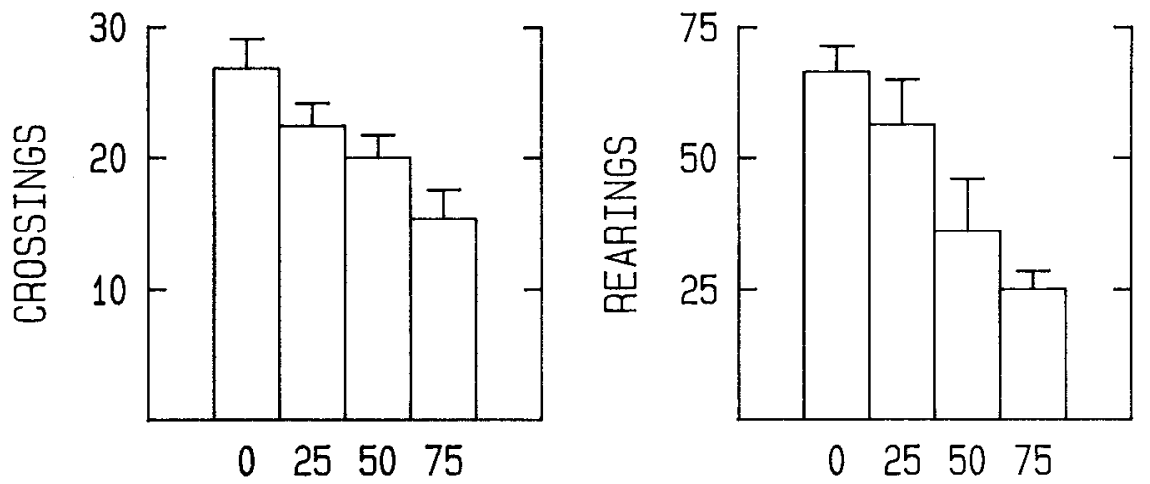

Figure l. Effects of BDP-12 on exploratory activity in a novel environment. Histograms show mean ( + SEM) number of centerline crossings and number of rearings after vehicle or three doses of BDP-12 (25,50, and $75 \mathrm{mg} / \mathrm{kg})$. Analysis of variance yielded significant main effects of the drug on crossings $[F(3,25)=4.11, p<0.02]$ and rearings $[F(3,25)=10.69, p<0.0001]$. Crossings were significantly fewer in rats injected with 75 $\mathrm{mg} / \mathrm{kg} \mathrm{BDP}-12$ compared to vehicle $(p<0.01)$. Rearings were significantly reduced after $50 \mathrm{mg} / \mathrm{kg}(p<0.01)$ or $75 \mathrm{mg} / \mathrm{kg}(p<0.001)$ injections compared to vehicle controls. There were no effects of drug treatment on the number of defecations during the 15 min. test $[F(3,25)=0.31, p>$ $0.5]$.

Nose-pokes in empty alleys were of no consequence and the trial was terminated after $40 \mathrm{sec}$ if no correct or incorrect responses were made. In these and all subsequent trials, only one correct or incorrect response (nose-poke) was allowed per trial.

Rats were trained to a criterion of three consecutive sessions in which each animal made eight correct responses in the last 10 trials. After criterion was reached the next session was carried out in the same manner except that following the fifth correct response trial, a fixed ratio 2 (FR2) reinforcement schedule was instituted and water delivery was doubled (to $300 \mu \mathrm{l}$ ) on the rewarded trials. The flashing light after incorrect responses was also on a FR2 schedule after the first five incorrect responses. In the next session, the first five correct or incorrect trials had FR1 schedules; thereafter, both correct and incorrect responses were reinforced independently at FR4 (with four times the water delivered on correct response trials; $600 \mu \mathrm{l}$ ). Each animal was trained one or two sessions of 20 trials each.

Training on the second odor pair proceeded with one or two sessions with 20 trials at FR1 followed by a session at FR4 (after the first 5 trials). The third odor pair was treated similarly. Training on the fourth, fifth, and all subsequent pairs were conducted with sessions at either FR1 $(n=8)$ or FR4 $(n=12)$ (after the first five rewarded trials in each session).

Retention testing. After all rats reached criterion on the fifth odor pair, preparations for drug testing commenced. First, the rats were retrained in one session on odor pair five. One or two days later they were given a retention test for odor pair five. This consisted of a five trial session in which neither correct nor incorrect responses were reinforced. The rats were then retrained in one session on odor pair five. On subsequent days, the rats were given retraining sessions on odors already learned, training on new odor pairs, and retention tests.

Drug testing. Drug test sessions were run in blocks of two consecutive days each. On the first day, half of the animals received intraperitoneal injections of BDP- $12(30 \mathrm{mg} / \mathrm{kg}$ in cyclodextrin) and the other half received vehicle (cyclodextrin) injections $10 \mathrm{~min}$ prior to training. On the second day, animals receiving drug on the first day were given vehicle injections and rats receiving vehicle on day one received a drug injection. One group of rats $(n=6)$ was given acquisition sessions containing three trials; these rats were tested twice after drug injections on different days and twice after vehicle injections on different days. One group of rats $(n=6)$ was given acquisition sessions with five trials and three tests each after vehicle and drug injections, all on different days. The final group $(n=8)$ was given five-trial acquisition sessions and the rats were tested four times after vehicle and drug injections. All acquisition trials were run on a FR1 reinforcement schedule. Odors assigned as positive and negative were partially counterbalanced across rats. Retention was tested one to three days after training with a series of five unrewarded probe trials.

Performance tests. To test drug effects on performance variables, the rats were retrained on an odor pair previously well-learned. They then received blocks of two consecutive days in which they were given retention tests for that odor pair after BDP-12 or vehicle injections.

\section{Results}

Effects on sponlaneous locomolor activily

The effects of BDP-12 on arousal levels were assessed by measuring exploratory activity of rats placed in a novel environment. The drug produced a dose-dependent reduction in both centerline crossings and number of rearings (Fig. 1). These effects were statistically significant for rearings at $50 \mathrm{mg} / \mathrm{kg}(p<0.01)$ and $75 \mathrm{mg} / \mathrm{kg}(p<0.001)$ and for line crossings at $75 \mathrm{mg} / \mathrm{kg}$ $(p<0.01)$. None of the rats exhibited seizures at any of the dosages tested and, conversely, there was no evidence of druginduced sleep. From these data, it appears that BDP-12 does not have stimulant-like effects and at lower dosages has only marginal effects on arousal.

\section{Performance on a balance beam test}

The balance beam task was used to test if the AMPA receptor modulator affects two classes of variables: (1) sensori-motor coordination and (2) motivation. With regard to the second point, the rats were trained to run back and forth across the narrow beam to obtain water rewards in the variant of the task used in these studies. In addition, it can be assumed that anxiety levels are a factor in performance, given that the beam was located 1 $m$ above the cushioned floor.

Figure 2 illustrates the results. The left-hand panels summarize the data for the mean time to cross the beam for two blocks of 10 traversals each. As shown, there was little between-rat ( $n$ $=8$ ) variance and performance was stable across blocks. There were no detectable differences in crossing time between vehicleinjection versus drug-injection days; thus, BDP-12 even at 50 $\mathrm{mg} / \mathrm{kg}$ does not appear to disturb sensori-motor coordination. The right hand panels summarize the results with regard to the number of crossings made within a 10 min session. The rats had the same scores following injections with BDP-12 at $30 \mathrm{mg} / \mathrm{kg}$ as they did after vehicle injections; however, $50 \mathrm{mg} / \mathrm{kg}$ of the drug produced a substantial depression in the number of crossings (paired $t(7)=2.69, p<0.05$ ). The AMPA receptor modulator at $50 \mathrm{mg} / \mathrm{kg}$ thus appears to suppress the frequency of over-trained responses as well as unlearned species-specific responses (e.g., rearings; see Fig. 1).

\section{Performance involving previously learned odors}

Partial reinforcement during initial odor discrimination training made it possible to use a retention test consisting of multiple 

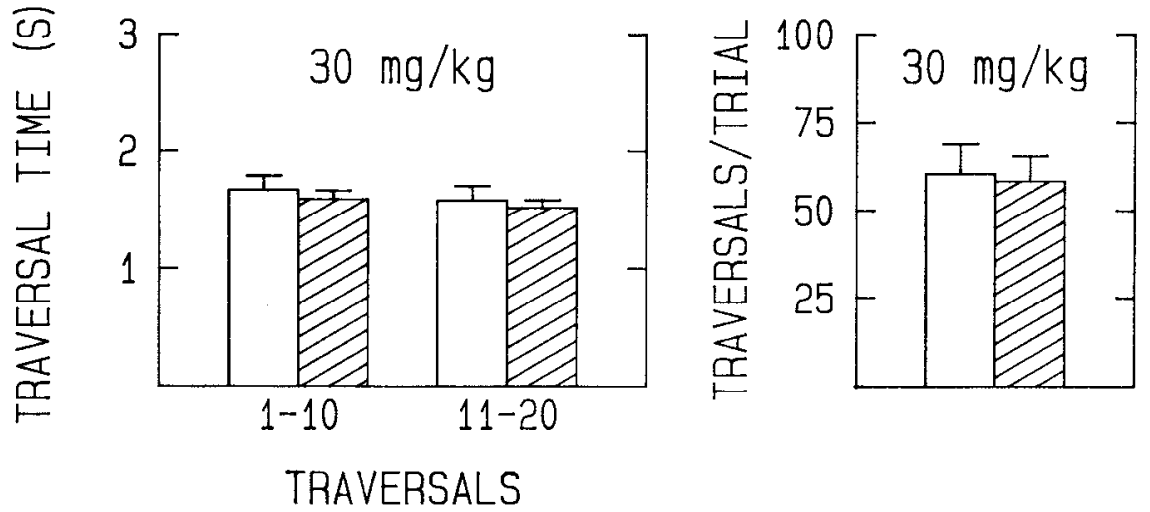

Figure 2. Effects of BDP-12 on performance of a balance beam task. Histograms show the mean $(+$ SEM) time spent crossing the beam for the first (1$10)$ and second $(11-20)$ block of 10 traversals after two doses of BDP-12 (hatched bars) or vehicle (open bars) as well as the total number of traversals made per $10 \mathrm{~min}$ trial.
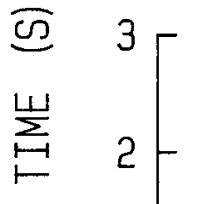

$\frac{1}{0}$
$\frac{1}{4}$
$\frac{1}{\square}$
$\frac{1}{1}$
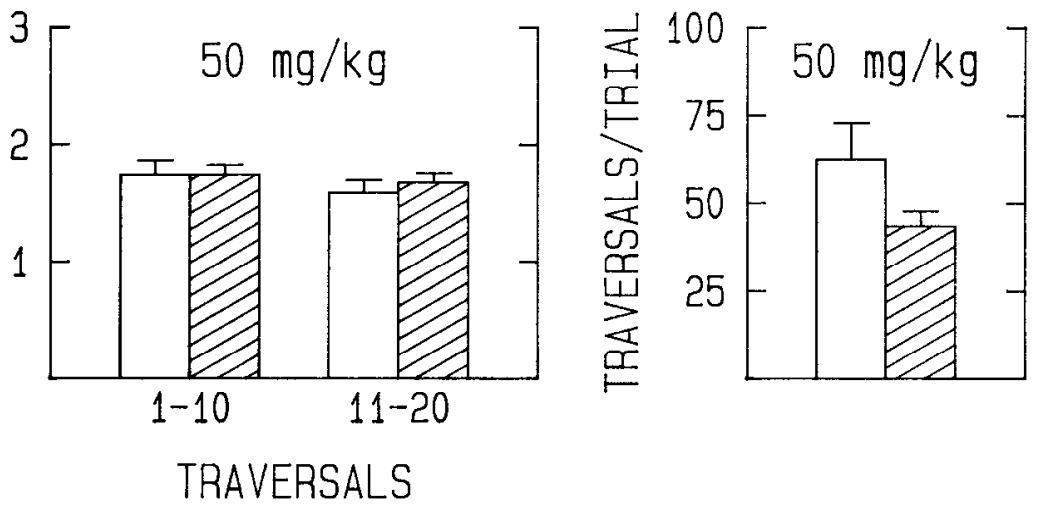

unrewarded trials to measure retention and performance variables while avoiding response extinction. The 20 rats averaged $96.0 \%( \pm 1.8 \%)$ correct responses in the five trial retention test carried out $24 \mathrm{hr}$ after retraining on a previously well-learned discrimination (odor pair five). Sixteen rats showed perfect responding, one rat made one response to the negative odor, and three rats failed to respond to either odor on one trial of the test.

The rats used in evaluating drug influences on acquisition and retention were also tested for drug effects on performance using a five trial retention test (unrewarded trials) for a previously acquired discrimination. Each animal was given drug and vehicle on the $2 \mathrm{~d}$ following retraining on an already-learned pair of odors. Nineteen rats were injected with BDP-12 at $30 \mathrm{mg} / \mathrm{kg}$; eight of these were then retested on another odor pair with the drug at $45 \mathrm{mg} / \mathrm{kg}$. Figure 3 (left) summarizes the percent correct responses (five unrewarded trials) for drug versus vehicle days. There was no evident drug effect on accuracy at $30 \mathrm{mg} / \mathrm{kg}$ but at $45 \mathrm{mg} / \mathrm{kg}$, performance was significantly impaired (paired $t(7)$ $=2.76, p<0.05$ ). Trial-by-trial analysis indicated that this effect was due to both an increase in the number of occasions on which the rats failed to respond and an increase in the number of incorrect choices. Figure 3 (right) also summarizes the group data for the latencies to make a correct response. There was a tendency for the animals to take longer to reach the reward port on drug injection days but this did not approach statistical significance even at $45 \mathrm{mg} / \mathrm{kg}$ (paired $t(7)=1.67, p>0.1$ ). This pattern of results resembles that observed in the balance beam task. That is, $30 \mathrm{mg} / \mathrm{kg}$ dosages had no detectable effects on performance; higher dosages (45-50 $\mathrm{mg} / \mathrm{kg}$ ) reduced responding without affecting the time taken to execute a response.

\section{Retention of olfactory cues}

Each of the 20 well-trained rats in the odor discrimination task was given two to four acquisition sessions with novel odor pairs
Figure 3. Performance on retention trials for previously learned odor pairs after vehicle or BDP-12 administration. Left panel, mean percent correct responses after two doses of BDP-12. Right panel, mean latency to respond on correct choices after two doses of BDP-12.
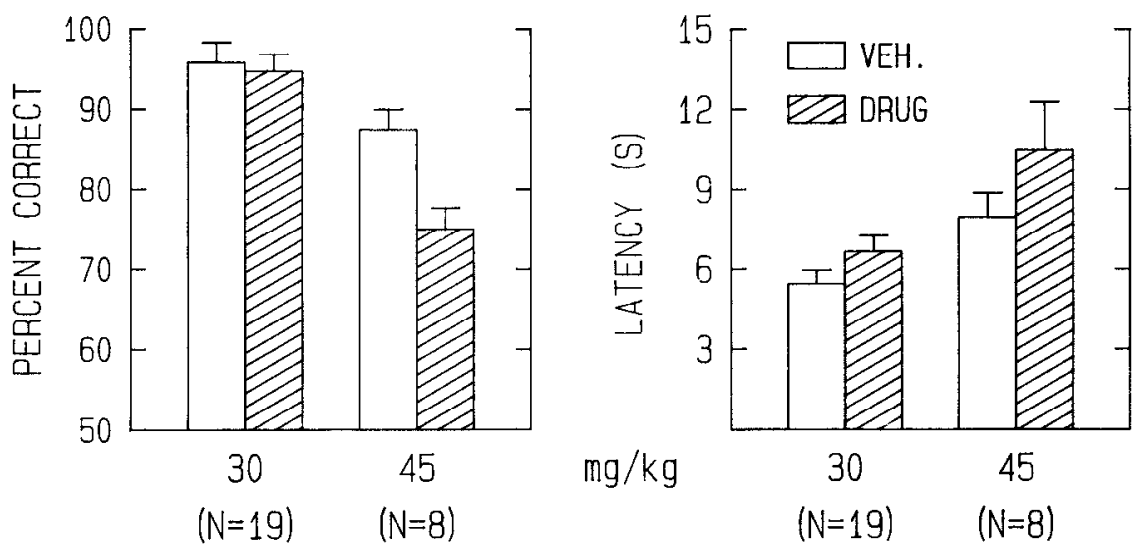


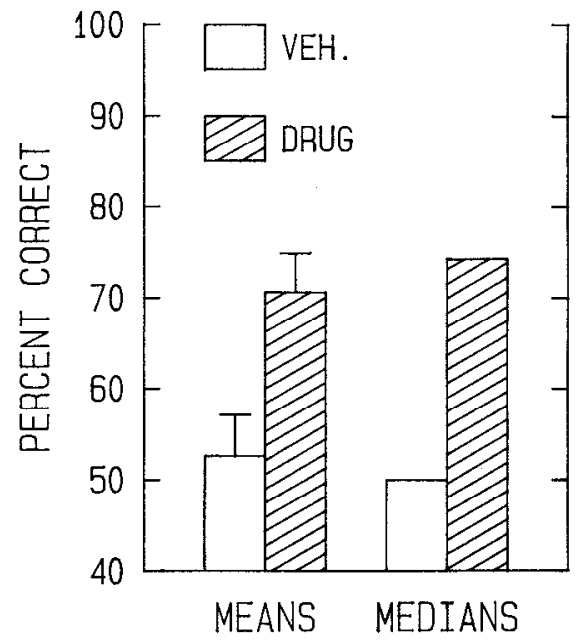

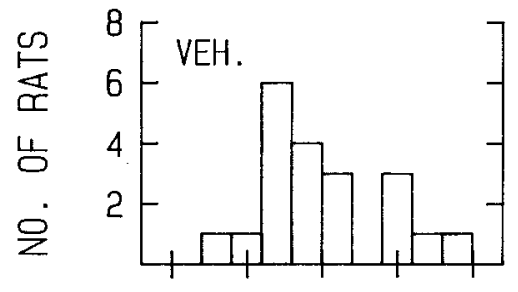

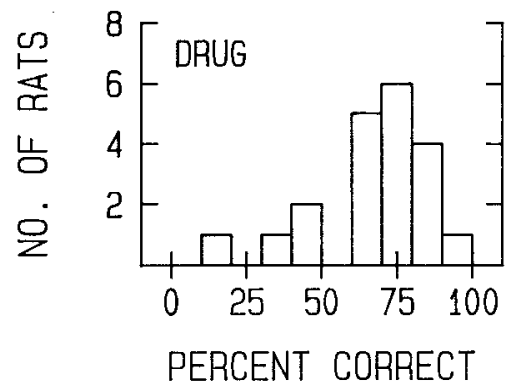

Figure 4. Retention performance after training with vehicle versus BDP12 injections. Left panel, histograms show mean $(+\mathrm{SEM})$ and median percent correct responses under the two training conditions. Chance performance is $50 \%$ correct. Right panel, frequency distributions show the number of rats having indicated mean percent correct scores for odor pairs trained after vehicle (VEH., top) and BDP-12 injections (DRUG, bottom). after BDP-12 (30 mg/kg) or vehicle injections. Retention was tested 24-72 hr. later in a session of five unrewarded trials. Figure 4 shows the retention scores for the 20 rats for the vehicletraining and drug-training sessions. When the rats were trained (three or five acquisition trials) after vehicle injections, average retention scores in test sessions $24-72$ hr later were not significantly different from chance $(52.6 \pm 4.5 \%$ correct $)$; the same rats trained after BDP-12 injections showed significantly higher retention scores $(70.6 \pm 4.2 \%$ correct; paired $t(19)=3.39, p<$ $0.01)$. Frequency distributions for percent correct responding are also shown in Figure 4. Five of the 14 rats given five training trials with vehicle had average scores of $75 \%$ or better across retention trials; none of the six animals given three training trials with vehicle had average retention scores above $60 \%$. This suggests, in accord with previous studies (e.g., Slotnick, 1994), that five trials is near threshold for the encoding of stable memory. Four of the rats had average retention scores at or below chance for odor pairs acquired under the influence of BDP-12; two of these animals were from the three training trial group. It remains to be determined if the dosage $(30 \mathrm{mg} / \mathrm{kg})$ of the AMPA receptor modulator used in the present study is optimal for enhancing retention.

The retention scores obtained for drug trials did not appear to be equal to those recorded when the rats had been given a large number (20 or more) of training trials under control conditions (e.g., see Fig. 3). This raises the question of how much additional training under control conditions would be needed to produce retention scores equivalent to those obtained with three to five acquisition trials under the influence of the drug. To provide an estimate of this, an additional group $(n=9)$ of well-trained rats was given 10 training trials under the same protocols described above. These animals had average retention scores of $79.3 \pm 4.5 \%$; that is, values similar to those recorded for the drug-treated rats. This result suggests that $\mathrm{BDP}-12$ reduces by about half the amount of training needed for a given strength of memory.

Median latencies to respond to the correct odor were obtained for each rat across the several drug-free retention sessions (five nonrewarded trials per session). The distribution of these scores for sessions involving odors trained 24-72 hr earlier on drug injection days was different from that for odors trained after vehicle injections. Figure 5 shows the number of rats with median latencies of less than $4 \mathrm{sec}, 4-6 \mathrm{sec}$, and greater than $6 \mathrm{sec}$. About equal numbers of rats fell into each of these bins for control odors; however, $80 \%$ of the group had median latencies of 4-6 sec for odors trained after injections of BDP-12 ( $p<$ $\left.0.01, \chi^{2}\right)$. In terms of individuals, five of seven rats that responded slowly $(>6 \mathrm{sec})$ during retention tests for control odors re-

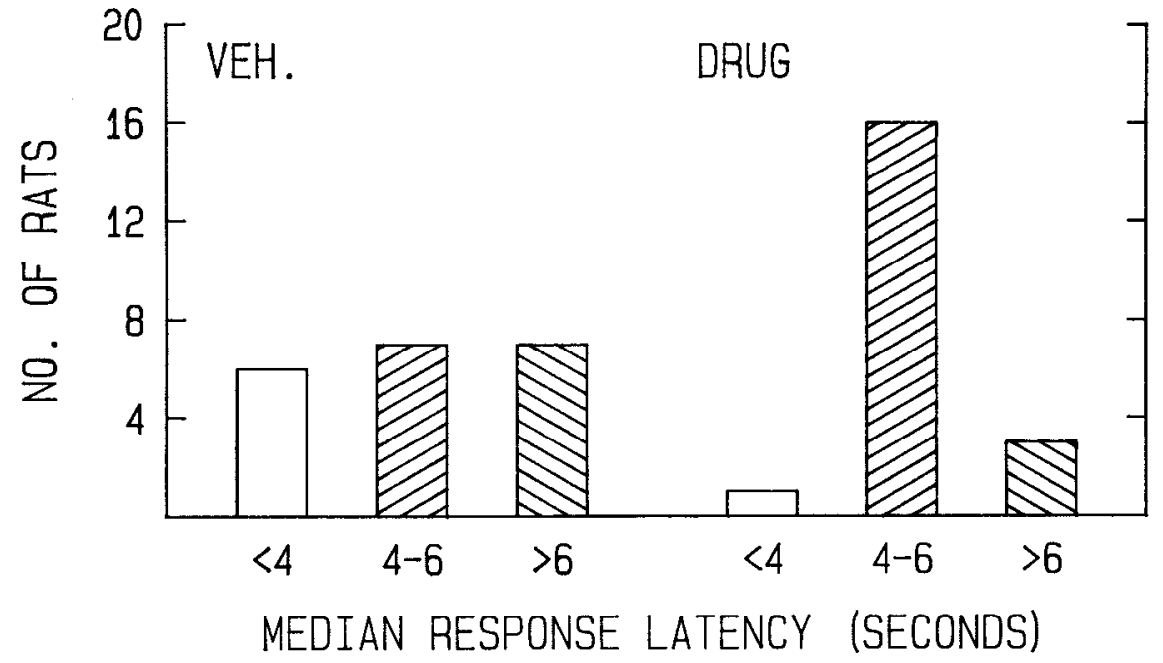

Figure 5. Response latencies during retention trials. Histogram shows frequency distributions for mediąn response latency on correct response trials for odors trained after vehicle or BDP-12 injections. 
Figure 6. Acquisition performance after vehicle or BDP-12 injections. Left panel, histograms show mean (+SEM) and median percent correct responses after the first choice trial during training on novel odors. Right panel, frequency distributions of mean correct responses during acquisition trials for the same 14 rats after vehicle (VEH. top) and BDP-12 (DRUG, bottom) injections.
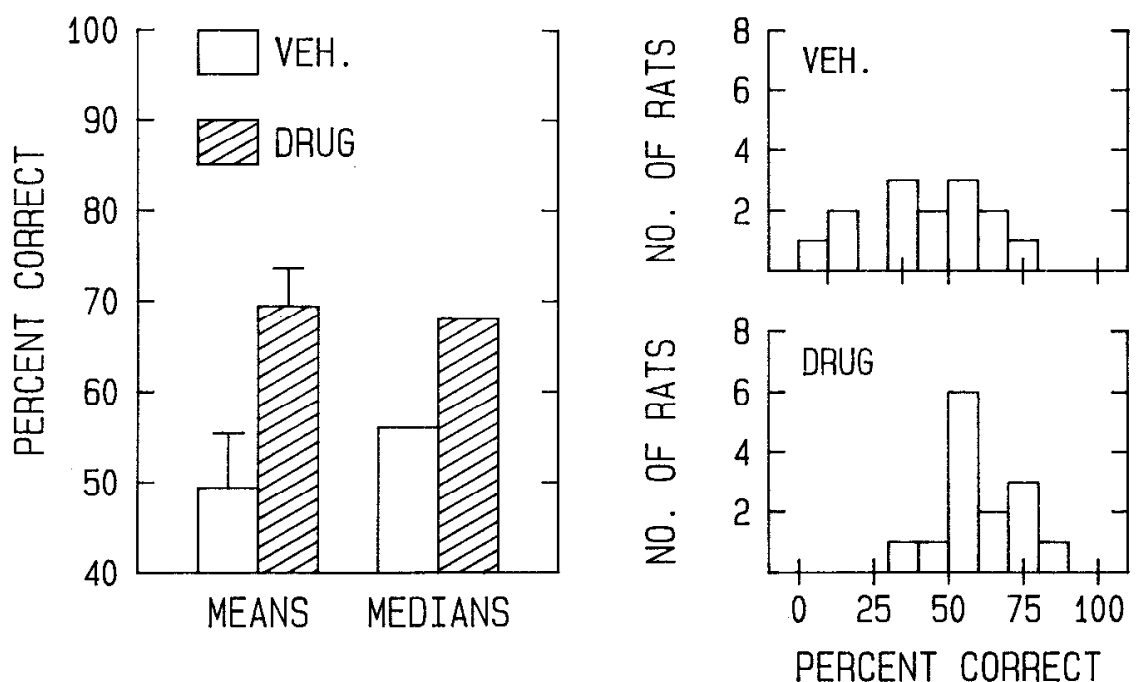

acted more quickly during sessions for odors trained under the drug $(-1.97 \pm 1.22 \mathrm{sec}, n=7)$ while five of six of those which reacted quickly $(<4 \mathrm{sec})$ to control odors were slower for drugtrained odors $(+0.95 \pm 0.57 \mathrm{sec})$. Seven of the rats had median latencies hetween 4 and $6 \mathrm{sec}$ to the control odors; these animals had equivalent latencies for drug-trained odors $(+0.10 \pm 0.26$ $\mathrm{sec}$ ). It is noteworthy that these seven animals had higher percent correct scores during the retention tests for control odors than did those responding more quickly or more slowly $[3.6 \pm 0.3$ correct responses in five trials vs $2.3 \pm 0.3$ (rapid responses, $t(11)=2.69, p<0.05$ ) and vs. $2.0 \pm 0.2$ (slow responses, $t(12)$ $=3.96, p<0.01)]$. This result suggests that learning which of the odors were correct during training affected response patterns in retention tests carried out after a considerable delay. That the rats were more likely to exhibit this pattern for odors trained after BDP-12 injections reinforces the conclusion that the drug enhanced learning.

\section{Acquisition of olfactory cues}

Figure 6 shows the mean and median percent correct responses during acquisition sessions for the 14 rats receiving five training trials; each rat was tested on three or four odor pairs after injections of drug or vehicle. The first choice trial and trials in which no response was made were eliminated from analysis. Scores for the training trials on vehicle injection days were at chance levels $(49.4 \pm 6.0 \%)$ but on drug injection days were significantly higher $(69.4 \pm 4.2 \%$; paired $t(13)=2.82, p<$ 0.02 ). Frequency distributions for the percent correct responding for these 14 rats are also shown in Figure 6 . Acquisition scores for vehicle and drug conditions were not correlated across rats $(r=+0.07, p>0.1)$. However, within-rat differences between drug versus vehicle acquisition sessions were correlated with the within-rat differences in retention scores $(r=+0.70, p<0.01)$. Thus, behavioral changes occurring in the presence of BDP-12 were reflected in later tests carried out in its absence.

The average latency to respond to the correct odor was 5.4 $\pm 0.5 \mathrm{sec}$ (mean $\pm \mathrm{SEM}$ ) on vehicle injections days and $5.5 \pm$ $0.5 \mathrm{sec}$ on drug injection days. One rat was considerably slower after BDP-12 injections ( $+5.3 \mathrm{sec}$ slower, average of four odor pairs with drug and four pairs with vehicle). Excluding this case, the drug versus vehicle latencies were correlated across rats ( $r$ $=+0.82, p<0.001$ ) suggesting that BDP-12 had little effect on the sampling-response tendencies of individual rats. Within- rat comparisons indicated that latencies during training sessions on drug or vehicle days did not correlate with latencies in the retention tests conducted $1-3 \mathrm{~d}$ later.

\section{Discussion}

The present study used olfactory learning to test the hypothesis that drugs which enhance synaptic currents mediated by AMPAtype glutamate receptors would facilitate the encoding of longterm memory. After extensive training on a series of odor discriminations, rats were given a suboptimal number of trials involving novel discriminations. Each rat was tested two to four times under both control and drug conditions, allowing for paired comparisons within each subject. Tests 1-3 d posttraining showed that when the rats were trained under the vehicle condition their retention performance was not different from chance, indicating that they learned little about the odor cues during training. Training after BDP- 12 injections, however, resulted in a significant improvement in retention performance. Measures of response latencies during the retention trials also indicated that the rats behaved differently to odor pairs that they had experienced on drug injection versus vehicle injection days.

The results also provide evidence that AMPA receptor modulators facilitate acquisition and that this effect is related to the enhancement of long-term memory. Specifically, BDP-12 was found to increase the number of correct responses made during five acquisition trials separated by 1 min intervals; moreover, the within-rat improvement in acquisition (drug versus vehicle) was correlated with the within-rat improvement in retention. It remains to be determined if AMPA receptor modulators also interact with any consolidation events that follow the acquisition sessions. With respect to the hypothesis that BDP-12 facilitates acquisition by facilitating LTP induction, it is not clear that posttraining injections would be efficacious. However, it would be interesting to test this in future work.

The enhancing effects of the benzoyl-piperidine compound on memory encoding are not likely to be due to changes in arousal or motivation. The drug was used in a common test of stimulantinduced arousal. At the dosage used in the olfactory learning paradigm, BDP-12 had little if any effect on exploratory activity, behaviors that are markedly increased by many central nervous system stimulants such as amphetamines, caffeine, cocaine, etc. (c.g., Swcrdlow et al., 1986; Nchlig ct al., 1992). Morcover, the same dosage did not detectably change any aspect of behavior 
in the balance beam task. It also left unaffected a variety of behaviors in the olfactory task. Rats given the drug prior to a retention test had about the same response latencies as they did when injected with vehicle; this would not have been expected if the compound had heightened arousal, increased motivation, facilitated olfactory processing, or enhanced the linkages between olfactory perception and learned responses. It is also noteworthy that BDP-12 at a dose which enhanced memory did not measurably alter response latencies during rewarded acquisition trials and left intact the individual differences between rats on this measure. While reinforcing the conclusion that moderate doses of the drug do not significantly change generalized state variables, these latter results also suggest that they have little effect on the extent to which rats attend to novel cues or react to reinforcement.

At higher dosages, BDP-12 did alter several performance measures. Exploratory activity was depressed in a dose-dependent fashion with higher drug levels causing substantial $(50 \%)$ changes. Higher dosages also caused a reduction in the number of crossings made in the balance beam task and depressed performance on retention trials in the olfactory paradigms; they did not, however, alter the time needed to traverse the heam or significantly increase the latency to respond to a positive cue. This pattern of results suggests that the drug produces a depression of learned and unlearned (e.g., rearings) prepotent responses without disturbing sensori-motor processing. The reasons why a compound that facilitates glutamatergic transmission would produce such effects are not obvious. However, it is known that damage to any of a variety of forebrain areas including frontal cortex and hippocampus causes increases in exploratory activity and the frequency of learned responses in rats ( $O$ 'Keefe and Nadel, 1978; Kolb, 1984, for reviews); possibly, then, high concentrations of BDP-12 result in excessive activity in polysynaptic networks in telencephalon (see below) that have inhibitory effects on behavior. A subtle version of such an effect at lower dosages might conccivably facilitatc acquisition by, for example, reducing extraneous behavior; however, this would presumably manifest itself as a reduced latency to respond to cues and, as noted, this was not detected in either acquisition or retention trials.

Given the above points regarding selectivity, it is not unreasonable to assume that the enhancing effect of BDP-12 on longterm memory is due to actions on brain events important to learning but not on behavioral/physiological variables (e.g., arousal, responsivity, and motivation) that secondarily influence. learning. Well-trained rats in the two-odor discrimination paradigm have learned that novel olfactory cues belong to a class pertinent to the testing situation and, hence, clearly attend to the novel odors during acquisition sessions. Under these circumstances, even a modest facilitation of excitatory transmission could have the effect of amplifying the signal being processed through olfactory cortex and on into deeper brain structures including hippocampus, amygdala, and frontal cortex. This effect could be quite large in such complex networks even with threshold levels of drug. Recent in vitro work has shown that the facilitatory actions of BDP-12 at individual synapses accumulate across the serial steps of the polysynaptic intra-hippocampal circuit; that is, the increase of the field EPSP relayed through dentate gyrus and the CA3 region to the field CA1 was more than threefold greater than that obtained with the monosynaptic CA3CA1 response. Moreover, even partial suppression of GABAergic transmission greatly augmented the differential effect of the drug on polysynaptic versus monosynaptic potentials (Sirvio et al., 1994). It is of interest that these latter results were obtained at concentrations of BDP-12 at or below the blood levels produced by the intraperitoneal injections $(30 \mathrm{mg} / \mathrm{kg})$ used in the present study. Since PET scan studies indicate that the bloodbrain barrier is freely permeable to the benzoyl-piperidine family to which BDP-12 belongs (Staubli et al., 1994b), it seems likely that polysynaptic effects of the kind observed in vitro also occur in vivo. A recent study has demonstrated that $30 \mathrm{mg} / \mathrm{kg}$ i.p. injections of BDP-12 do not generally alter cell discharge rate of hippocampal neurons but do change the nature of their discharge patterns in relation to behavior in a short-term memory task (Granger et al., in press). These results suggest that the drug dosage used in the olfactory learning experiments has electrophysiological effects in the hippocampus.

In addition to facilitating communication in serial networks selectively driven by attentive sampling of novel cues, AMPA receptor modulators might also enhance memory by a more direct action on encoding processes. As noted, the drugs promote the induction of LTP in vitro (Arai and Lynch, 1992; J. Larson, P. Xiao, and G. Lynch, unpublished observations) and in vivo (Staubli et al., 1994b); accordingly, the behavioral results reported here are as predicted from the much discussed hypothesis that the potentiation effect is the substrate of many commonplace forms of memory. It is noteworthy that chronic recording experiments indicate that the influence of benzoyl-piperidine drugs on LTP is much greater than anticipated from their actions on monosynaptic responses. Specifically, the drugs caused a more than two-fold increase in potentiation induced by suboptimal stimulation while facilitating synaptic potentials by approximately $20 \%$ (Staubli et al., 1994b). At threshold, then, increases in fast, depolarizing currents may have nonlinear effects on the slower, voltage-dependent responses of NMDA receptors. While in vitro studies are needed to test this point, the observed disproportionate actions of benzoyl-piperidine drugs on LTP versus synaptic transmission suggest one factor that could contribute to their selective effects on memory encoding.

Finally, the influences of AMPA receptor modulators on polysynaptic communication and on the induction of LTP are likely to be synergistic. That is, responses in the later segments of an active network series will be more likely to potentiate because of the local effects of the drugs and because they are augmented to a substantial degree by the actions of the drugs at all prior steps in the series. In this way, drug concentrations so low as to produce marginal effects at individual contacts could have significant influence on plasticity in complex networks. Since such networks presumably underlie telencephalic memory, this argument provides a possible explanation for why dosages of BDP-12 that do not detectably alter overt behavior nonetheless accelerate learning.

\section{References}

Arai A, Lynch G (1992) Factors regulating the magnitude of long-term potentiation induced by theta pattern stimulation. Brain Res 598:173184.

Arai A, Kessler M, Xiao P, Ambros-lngerson J, Rogers G, Lynch G (1994) A centrally active drug that modulates AMPA receptor gated currents. Brain Res 638:343-346.

Bliss TVP, Collingridge GL (1993) A synaptic model of memory: longterm potentiation in the hippocampus. Nature 361:31-39.

Eichenbaum H, Fagan A, Cohen NJ (1986) Normal olfactory discrimination learning set and facilitation of reversal learning after combined and separate lesions of the fornix and amygdala in rats: implications for preserved learning in amnesia. J Neurosci 6:1876-1884. 
Granger R, Staubli U, Davis M, Perez Y, Nilsson L, Rogers GA, Lynch G (1993) A drug that facilitates glutamatergic transmission reduces exploratory activity and improves performance in a learning-dependent task. Synapse 15:326-329

Granger R, Deadwyler S, Davis M, Moskovitz B, Rogers R, Lynch G (in press) Facilitation of glutamate receptors reverses an age-associated memory impairment in rats. Synapsc, in press.

Ito I, Tanabe S, Kohda A, Sugiyama $\mathbf{H}$ (1990) Allosteric potentiation of quisqualate receptors by a nootropic drug aniracetam. J Physiol (Lond) 424:533-543.

Kolb B (1984) Functions of the frontal cortex of the rat: a comparative review. Brain Res Rev 8:65-98.

Lu X-CM, Slotnick BM (1990) Acquisition of an olfactory learningset in rats with lesions of the mediodorsal thalamic nucleus. Chem Senses 15:713-724.

Nehlig A, Daval J-L, Debry G (1992) Caffeine and the central nervous system: mechanisms of action, biochemical, metabolic and psychostimulant effects. Brain Res Rev 17:139-170.

O'Keefe J, Nadel L (1978) The hippocampus as a cognitive map, Oxford: Oxford UP.

Rogan M, Staubli U, LeDoux JE (1994) Facilitation of AMPA receptors accelerates classical fear conditioning in rats. Soc Neurosci Abstr 20:1007.

Shors TJ, Servatius RJ, Thompson RF, Rogers G, Lynch G (1994)
Facilitation of classical conditioning through enhanced glutamatergic neurotransmission. Neurosci Lett 186:1-4.

Slotnick BM (1994) The enigma of olfactory learning revisited. Neuroscience $58: 1-12$.

Slotnick BM, Katz HM (1974) Olfactory learning-set formation in rats. Science 185:796-798.

Slotnick BM, Risser JM (1990) Odor memory and odor learning in rats with lesions of the lateral olfactory tract and mediodorsal thalamic nucleus. Brain Res 529:23-29.

Slotnick BM, Kufcra A, Silberberg AM (1991) Olfactory learning and odor memory in the rat. Physiol Behav 50:555-561.

Staubli U, Fraser D, Faraday R, Lynch G (1987) Olfaction and the "data" memory system in rats. Behav Neurosci 101:757-765.

Staubli U, Thibault O, DiLorenzo M, Lynch G (1989) Antagonism of NMDA receptors impairs acquisition but not retention of olfactory memory. Behav Neurosci 103:54-60.

Staubli U, Rogers G, Lynch G (1994a) Facilitation of glutamate receptors enhances memory. Proc Natl Acad Sci USA 91:777-781.

Staubli U, Perez Y, Xu F, Rogers G, Ingvar M, Stone-Elander S, Lynch G (1994b) Centrally active modulators of glutamate (AMPA) receptors facilitate the induction of LTP in vivo. Proc Natl Acad Sci USA 91:11158-11162.

Youngentob SL, Markert LM, Hill TW, Matyas EP, Mozell MM (1991) Odorant identification in rats: an update. Physiol Behav 49:12931296. 